\section{Die Crux mit der Nahrungsmittelallergie}

N ahrungsmittelallergien sind für viele Betroffene und ihre Familien eine Crux. Auch Allergologen sind manchmal damit überfordert, den $\mathrm{Pa}$ tienten den Alltag mit der Allergie genau zu erklären: Wie kann man bestimmte Lebensmittel wirklich meiden, wie machen sich verborgene Kreuzallergien bemerkbar? In Allergieberatung geschulte Fachkräfte sind rar, das Internet enthält viel Irrationales. Hier kann der vorliegende Ratgeber eine kompetente Hilfe sein. Er ist verständlich geschrieben, bietet umfassende Informationen zu Nahrungsmittelallergien, Allergietests und enthält auch sogenannte Allergenporträts. Diese zeigen auf, in welchen Lebensmitteln die jeweiligen Allergene stecken, wie sie zu meiden sind und wie Mangelerscheinungen verhindert werden können.

Die erfahrenen und langjährig tätigen Autorinnen des Buches vermitteln laiengerecht und aktuell alles Wesentliche im weiten Feld der Nahrungsmittelallergien und -unverträglichkeiten. Natürlich kann ein Buch nicht das individuelle Gespräch ersetzen, aber speziell das vorliegende Werk vermag mit seinen wertvollen Informationen den Patienten-Kontakt „unterfüttern“"

Dr. F. Grüttner, Hannover

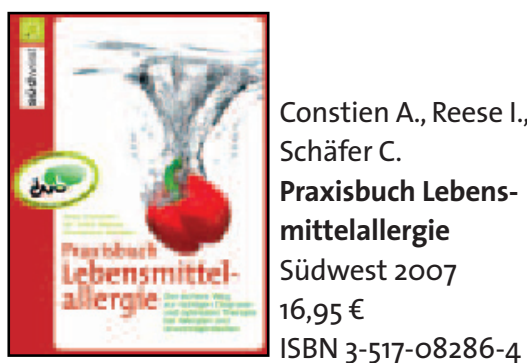

\title{
Essen muss Spaß machen - auch bei Nahrungsdmittelallergie
}

D ie größte Teil der erwachsenen Nahrungsmittelallergiker leidet unter pollenassoziierten Nahrungsmittelallergien. Führt lediglich der Genuss eines Apfels zu einem Kratzen im Hals, so lässt sich dieser meiden und durch andere Obstsorten ersetzen. Was aber, wenn diverse Nahrungsmittel zu Reaktionen führen und diese sich heftiger äußern als nur durch ein Halskratzen? Gerade wenn Asthmapatienten unter pollenassoziierten Nahrungsmittelallergien leiden, ist Unterstützung gefragt. Ein Kochbuch kann dabei nicht die ernährungstherapeutische Beratung betroffener Personen ersetzen, aber es stellt eine entscheidende Hilfe für den Erhalt der Lebensqualität im Alltag dar.

Mit dem „TRIAS-Kochbuch für Kreuzallergiker" ist den Diplom-Ökotrophologinnen Christiane Schäfer und Anne Kamp ein praxisrelevantes Buch gelungen. Der Schwerpunkt liegt auf den pollenassoziierten Nahrungsmittelallergien, aber auch andere Kreuzreaktionen wie z. B. bei Latexsensibilisierung werden angesprochen. Schon mit der Einführung werden die Autorinnen die meisten Patienten dort abholen, wo sie sich hingedrängt fühlen: Die häufige Bagatellisierung der
Erkrankung wird deutlich genannt und bemängelt. Ein Selbsttest hilft fraglich betroffenen Personen bei der Selbsteinschätzung. Kurzinformationen über die Leitallergene Beifuß, Birke, Gräser, Traubenkraut, Latex und Hausstaubmilbe enthalten eine $\mathrm{Zu}$ ordnung, welche Nahrungsmittel häufig bzw. selten Reaktionen auslösen oder aber gut vertragen werden. Sowohl Obstsorten als auch Kräuter und Gewürze werden im Hinblick auf ihre Allergenität bewertet.

Im Rezeptteil, der eine umfangreiche Sammlung an leckeren Vorschlägen zur Zubereitung von Frühstück, Kleinigkeiten, Hauptgerichten, Desserts sowie Kuchen und Gebäck enthält, sind die Autorinnen kreativ geworden: Alle Rezepte sind den Ampelfarben gemäß rot, gelb und grün bewertet worden, so dass Betroffenen ein Blick genügt, um feststellen, ob das jeweilige Rezept für sie geeignet ist. Darüber hinaus können gelbe Ampeln mit dem Warnhinweis „Aufgepasst - individuelle Unverträglichkeit möglich" durch Rezeptabwandlungen auf grün gestellt werden. Die Rezeptsammlung mit den dazugehörigen Ampeln ist im vorderen Buchrücken im Überblick dargestellt. Rote Stopp-
Ampeln finden sich nur dreimal. Zubereitungsalternativen sind auch für viele Rezepte mit grünen Ampeln genannt, was die Rezeptauswahl noch vergrößert. Verwirrend ist allerdings, dass auch diese Varianten in dem Kasten "So stellen Sie Ihre Ampel auf grün!“ stehen. Hier wäre es verständlicher gewesen, einen separaten Kasten mit Abwandlungstipps zu erstellen. Den Schluss bilden wichtige Adressen und Buchtipps sowie eine Empfehlung des Deutschen Allergie- und Asthmabundes (DAAB).

Auch mit ihrem zweiten Kochbuch für Personen mit Unverträglichkeitsreaktionen erfüllen die beiden Autorinnen hundertprozentig ihren Grundsatz „Essen muss unbedingt Spaß machen", ohne den Informationsteil zu kurz kommen zu lassen. Das Kochbuch für Kreuzallergiker setzt sich damit von vielen anderen Kochbüchern für Patienten mit Unverträglichkeiten deutlich ab.

Dr. Imke Reese, München

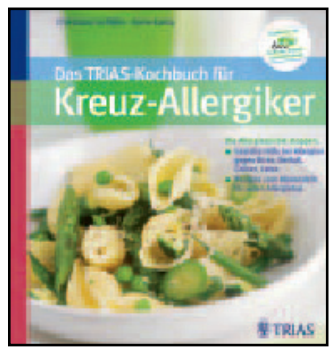

Schäfer C., Kamp A. TRIAS-Kochbuch für KreuzAllergiker TRIAS 2008 $19,95 €$ ISBN 9783830434399 